\title{
Hard-core Final State Interaction Effects in Deep Inelastic Scattering
}

\author{
Jaime Besprosvany ${ }^{1}$ and S. A. Gurvitz ${ }^{2,3}$ \\ ${ }^{1}$ Department of Physics of Complex Systems and ${ }^{2}$ Department of Particle Physics, \\ Weizmann Institute of Science, Rehovot 76100, Israel \\ ${ }^{3}$ TRIUMF, Vancouver, B.C., Canada V6T $2 A 3$
}

\begin{abstract}
Hard-core final state interaction effects in the response function are investigated in the asymptotic limit of momentum transfer $q \rightarrow \infty$. The relevant scattering contribution is displayed and a modification of the otherwise expected free response is obtained for Bose and Fermi systems. A comparison with other treatments of final state interactions is made.
\end{abstract}

To be published in Phys. Lett. A.

Typeset using REVTEX 
Important information on Bose and Fermi systems can be obtained from inclusive scattering of a weak probe in which energy $\omega$ and momentum $q$ are transferred to these systems. Such measurements in the large $q$ region offer the possibility to obtain the single-particle momentum distribution $n(p)$. Indeed, if for large $q$ the response function $S(q, \omega)$ approaches the impulse approximation (IA), then the momentum distribution can be readily obtained. In terms of the reduced response $\phi(q, \mathrm{y}) \equiv(q / m) S(q, \omega)$ the IA is given as

$$
\phi^{I A}(\mathrm{y})=\int \frac{d^{3} p}{(2 \pi)^{3}} n(p) \delta(\mathrm{y}-\hat{\boldsymbol{q}} \cdot \boldsymbol{p})=\frac{1}{2 \pi A} \operatorname{Re} \int d^{3} r d x \exp (i \mathrm{y} x) \rho_{1}(\boldsymbol{r} ; \boldsymbol{r}+x \hat{\boldsymbol{q}}),
$$

where $\mathrm{y}=m \omega / q-q / 2$ is a scaling variable, $A$ the number of particles in the system, $m$ the mass of the particle, and $\rho_{1}$ is the one-particle density matrix in coordinate space. In particular, an examination of Eq. (11) implies that the IA gives a distinctive delta function peak for the case of a momentum distribution containing a condensate fraction $n_{0}$, namely $n(p)=n_{n c}(p)+n_{c}(p)$, where $n_{c}(p)=n_{0}(2 \pi)^{3} \delta(\boldsymbol{p})$. However, final state interactions (FSI) can modify Eq. (1) and it is of special interest to study their effect on the condensate peak.

The treatment of FSI is a very complicated problem in general and it is desirable to study the response in the asymptotic limit $q \rightarrow \infty$, and $\mathrm{y}=$ const, where the problem is greatly simplified. Indeed, Eq. (ID) represents the lowest order term in a multiple scattering series (MSS), i. e., $\phi(q, \mathrm{y})-\phi^{I A}(\mathrm{y})=O(v / q)$, where $v$ is the potential. This means that the IA is exact in the asymptotic limit in the case of regular potentials. In addition, if the interactions contain a strong repulsive component (hard-core), only this part has to be taken into account in the asymptotic limit, while the regular part will not contribute.

In the following we treat the asymptotic response in terms of a MSS describing the FSI. Being able to sum over the MSS into simple analytical expressions, we investigate for both Bose and Fermi systems how the IA is modified in this limit. In particular, we explicitly show how the $\delta$-function condensate peak is smeared due to FSI. We also make a comparison with other approaches for the response function in the asymptotic limit, thus clarifying in this way the nature of the approximations underlying these.

The exact asymptotic expression for the Green's function entering the response in the 
case of hard-core FSI was obtained in Ref. [1] by use of the well-known result that the Schroedinger equation coincides with the equations of geometrical optics when the energy of the struck particle goes to infinity. The geometrical optics solution is known for a wave function describing hard-core scattering of the knock-on particle '1' off spectator particles, '2',.., 'A'. It is a plane wave describing particle '1', modified by a subtraction of "shadow" regions behind the scatterers and adding the reflected waves [2]. The resulting form for the response $\tilde{\phi}(\mathrm{y}) \equiv \lim _{q \rightarrow \infty} \phi(q, \mathrm{y})$ is

$$
\tilde{\phi}(\mathrm{y})=\frac{1}{A ! \pi} \operatorname{Re} \int d^{2} b_{1} d z_{1} d z_{1}^{\prime} d^{3} r_{2} \ldots d^{3} r_{A} \rho_{A} \theta\left(z_{1}^{\prime}-z_{1}\right) e^{i \mathrm{y}\left(z_{1}^{\prime}-z_{1}\right)} \prod_{i=2}^{A}\left(1-S_{1 i}\right)
$$

where

$$
S_{1 i}=\theta\left(a-\left|\boldsymbol{b}_{1}-\boldsymbol{b}_{i}\right|\right) \theta\left(z_{1}^{\prime}-z_{i}+w_{i}\right) \theta\left(w_{i}-z_{1}+z_{i}\right)
$$

with $w_{i}=\left(a^{2}-\left|\boldsymbol{b}_{1}-\boldsymbol{b}_{i}\right|^{2}\right)^{1 / 2}, \boldsymbol{r}_{1}=\left(\boldsymbol{b}_{1}, z_{1}\right), \boldsymbol{r}_{1}^{\prime}=\left(\boldsymbol{b}_{1}, z_{1}^{\prime}\right)$, and $a$ is the radius of the core. $\rho_{A}$ is the $A$-body density matrix defined as

$$
\rho_{A} \equiv \rho_{A}\left(\boldsymbol{r}_{1}, \boldsymbol{r}_{2}, \ldots, \boldsymbol{r}_{A} ; \boldsymbol{r}_{1}^{\prime}, \boldsymbol{r}_{2}, \ldots, \boldsymbol{r}_{A}\right)=A ! \Psi_{0}\left(\boldsymbol{r}_{1}, \boldsymbol{r}_{2}, \ldots, \boldsymbol{r}_{A}\right) \Psi_{0}^{*}\left(\boldsymbol{r}_{1}^{\prime}, \boldsymbol{r}_{2}, \ldots, \boldsymbol{r}_{A}\right)
$$

where $\Psi_{0}$ is the ground state wave function of the $A$-particle system, normalized in a box of volume $V$ as $\left\langle\Psi_{0} \mid \Psi_{0}\right\rangle=1$. The corresponding $n$-body density matrices are defined by:

$$
\rho_{n}\left(\boldsymbol{r}_{1}, \ldots, \boldsymbol{r}_{n} ; \boldsymbol{r}_{1}^{\prime}, \ldots, \boldsymbol{r}_{n}\right)=\frac{1}{(A-n) !} \int d^{3} r_{n+1} \cdots d^{3} r_{A} \rho_{A}\left(\boldsymbol{r}_{1}, \ldots, \boldsymbol{r}_{A} ; \boldsymbol{r}_{1}^{\prime}, \ldots, \boldsymbol{r}_{A}\right)
$$

Notice that the $\rho_{A}$ needed is off-diagonal in the coordinate of the struck particle $\boldsymbol{r}_{1}$, and diagonal in the coordinates of the spectator particles.

With its rather simple appearance, Eq. (2) gives the exact account for the FSI of the knock-on particle with spectators in the asymptotic limit. It is only the hard-core component of FSI which survives in this limit and modifies the free eikonal Green's function in Eq. (2) by the product of $\left(1-S_{1 i}\right)$. This product forbids propagation along the hard-core shadow configurations (the hatched region in Fig. 1). By expanding the product in the integrand (2) 


$$
\prod_{i=2}^{A}\left(1-S_{1 i}\right)=1-\sum_{i} S_{1 i}+\sum_{i>j} S_{1 i} S_{1 j}-\sum_{i>j>k} S_{1 i} S_{1 j} S_{1 k}+\cdots
$$

one gets parallelly for the response function

$$
\tilde{\phi}(\mathrm{y})=\sum_{i=1}^{A} \tilde{\phi}_{i}(\mathrm{y})
$$

where the first term, $\tilde{\phi}_{1}(\mathrm{y})$, corresponds to IA, Eq. (1) and the higher order terms represent a Glauber-type MSS, describing FSI of the knock-out particle with $A-1$ spectator particles.

As a first instructive example for the application of Eq. (2) we consider the asymptotic response of a system of $n_{0} A$ particles occupying the zero momentum state, as can be obtained from the Bose condensation phenomenon in an ideal gas. We take the $A$-body density-matrix in the form $\rho_{A}=\rho_{A c}^{(1)}=n_{0} A ! / V^{A}$, demanding $\rho_{1 c}\left(\boldsymbol{r}_{1} ; \boldsymbol{r}_{1}^{\prime}\right)=n_{0} \rho$, where $\rho=A / V$. By substituting $\rho_{A c}^{(1)}$ into Eq. (2), we notice that its independence of the particles' coordinates enables Eq. (2) to be written as products of equivalent integrals over the spectator coordinates:

$$
\tilde{\phi}_{c}^{(1)}(\mathrm{y})=\frac{n_{0}}{\pi A} \operatorname{Re} \int d z_{1} d z_{1}^{\prime} d^{2} b_{1} \rho \theta\left(z_{1}^{\prime}-z_{1}\right) \exp \left[i \mathrm{y}\left(z_{1}^{\prime}-z_{1}\right)\right]\left[1-\frac{1}{A} \rho \tilde{E}\left(z_{1}^{\prime}-z_{1}\right)\right]^{A-1},
$$

with

$$
\begin{array}{r}
\tilde{E}\left(z_{1}^{\prime}-z_{1}\right)=\int d^{2} b_{i} d z_{i} \theta\left(a-\left|\boldsymbol{b}_{1}-\boldsymbol{b}_{i}\right|\right) \theta\left(z_{1}^{\prime}-z_{i}+w_{i}\right) \theta\left(w_{i}-z_{1}+z_{i}\right) \\
=2 \pi \int_{0}^{a} d b_{1 i} b_{1 i}\left(z_{1}^{\prime}-z_{1}+2 \sqrt{a^{2}-b_{1 i}^{2}}\right)=\pi a^{2}\left(z_{1}^{\prime}-z_{1}+\frac{4 a}{3}\right)
\end{array}
$$

and $b_{1 i}=\left|\boldsymbol{b}_{1}-\boldsymbol{b}_{i}\right|$. The function $\tilde{E}$ depends only on $x=z_{1}^{\prime}-z_{1}$ and then Eq. (8) may be rewritten as

$$
\tilde{\phi}_{c}^{(1)}(\mathrm{y})=\frac{n_{0}}{\pi} \operatorname{Re} \int_{0}^{\infty} d x e^{i \mathrm{y} x}\left[1-\frac{1}{A} \rho \pi a^{2}\left(x+\frac{4 a}{3}\right)\right]^{A-1}
$$

By expanding the integrand in Eq. (10) using the binomial formula one explicitly reproduces the IA term and the MSS, Eq. (77). In the limit $A \rightarrow \infty$ Eq. (10) goes over to

$$
\tilde{\phi}_{c}^{(1)}(\mathrm{y})=\frac{n_{0}}{\pi} e^{\frac{-4 \pi \rho a^{3}}{3}} \operatorname{Re} \int_{0}^{\infty} d x \exp [i \mathrm{y} x-\rho \sigma x]=\frac{n_{0}}{\pi} e^{\frac{-4 \pi \rho a^{3}}{3}} \frac{\sigma \rho}{(\sigma \rho)^{2}+\mathrm{y}^{2}}
$$

where $\sigma=\pi a^{2}$. 
This result explicitly shows smearing of the $\delta$-function peak due to FSI, first suggested by Hohenberg and Platzman [3]. Notice that the $\delta$-function peak survives in the IA for finite $A$, and disappears only if $A \rightarrow \infty$. This explains why smearing of the condensate did not occur in a truncated MSS [4]. Our treatment implies that it is not possible to determine a finite number of collisions leading to the extinction of the singularity as imprecisely stated in Ref. [5].1 In addition, unlike Ref. [5], in our treatment the role of the hard-core in the smearing is specific to the asymptotic limit. However, at finite $q$ regular interactions can also produce the effect [6].

Eqs. (10), (11) give the asymptotic response for systems with a constant $\rho_{A}$ in space. Actually, such $\rho_{A}$ cannot be produced because of the presence of configurations with particles' cores interceptions (square-hatched region in Fig. 1). In fact, the resulting Lorentzian response in Eq. (11) violates sum rules, and in particular, $\int \tilde{\phi}_{c}^{(1)}(\mathrm{y}) d \mathrm{y} \neq n_{0}$, and in fact, the latter integral and $\int \tilde{\phi}_{c}^{(1)}(\mathrm{y}) \mathrm{y}^{2} d \mathrm{y}$ diverge. These problems are overcome by modifying the density-matrix $\rho_{A c}^{(1)}$, imposing the hard-core constraint. Specifically, we consider the $A$-body density-matrix

$$
\rho_{A c}^{(2)}=\rho_{A c}^{(1)} \eta C\left(\boldsymbol{r}_{i}, \boldsymbol{r}_{i}^{\prime}\right)
$$

where $C\left(\boldsymbol{r}_{i}, \boldsymbol{r}_{i}^{\prime}\right)=\prod_{i=2}^{A} \theta\left(r_{i 1}-a\right) \theta\left(r_{i 1}^{\prime}-a\right)$, and $r_{1 i}=\left|\boldsymbol{r}_{i}-\boldsymbol{r}_{1}\right|, r_{1 i}^{\prime}=\left|\boldsymbol{r}_{i}-\boldsymbol{r}_{1}^{\prime}\right|$. Here the normalization factor $\eta$ is included, in order to to satisfy the condition for the one-body density $\rho_{1 c}=n_{0} \rho$, Eq. (5). We obtain

$$
\eta(x)=(1-u(x) / V)^{1-A}
$$

\footnotetext{
${ }^{1}$ Since the main contribution of the integral in Eq. (11) comes from any interval $[0, \bar{x}]$ with $\bar{x}>>1 /(\rho \sigma)$, the response could be approximated by contributions from a finite number of collision terms $N \sim \rho \sigma \bar{x}$ (replacing $e^{-\rho \sigma x}$ in the integrand in Eq. (11) by its Taylor expansion to $N$ th order). However, contributions from the interval $[\bar{x}, \infty]$ remain singular unless one takes $N \rightarrow \infty$ which corresponds to considering an infinite number of collisions.
} 
where

$$
u(x)=\frac{8}{3} \pi a^{3}+\left(\pi a^{2} x-\frac{1}{12} \pi x^{3}-\frac{4}{3} \pi a^{3}\right) \theta(2 a-x) .
$$

Configurations with particles' core interception for the hit and spectator particles, (Fig. 1), are actually excluded automatically by the $\left(1-S_{1 i}\right)$ factors in Eq. (2). Explicitly, when the latter Eq. is evaluated by substituting into it $\rho_{A c}^{(2)}$ given in Eq. (12), the factor $C\left(\boldsymbol{r}_{i}, \boldsymbol{r}_{i}^{\prime}\right)$ is redundant. For this density-matrix it immediately follows

$$
\tilde{\phi}_{c}^{(2)}(\mathrm{y})=\frac{n_{0}}{\pi} e^{\frac{-4 \pi \rho a^{3}}{3}} \operatorname{Re} \int_{0}^{\infty} d x \exp [i \mathrm{y} x-\rho(\sigma x-u(x))]
$$

The behavior of $\tilde{\phi}_{c}^{(2)}(\mathrm{y})$, being other than Lorentzian at large $|\mathrm{y}|$, allows for the (partly asymptotic) sum rules to be satisfied. Indeed one obtains from Eq. (15)

$$
\begin{aligned}
\int d \mathrm{y} \tilde{\phi}^{(2)}(\mathrm{y}) & =n_{0} \\
\int d \mathrm{y} \tilde{\phi}^{(2)}(\mathrm{y}) \mathrm{y} & =0 \\
\int d \mathrm{y} \tilde{\phi}^{(2)}(\mathrm{y}) \mathrm{y}^{2} & =\frac{2 m}{3}<K>
\end{aligned}
$$

where

$$
<K>=-\left.\frac{1}{2 m} \frac{d^{2}}{d x^{2}} \rho_{1}^{(2)}(0, x)\right|_{x \rightarrow 0}
$$

is the average kinetic energy.

At low densities or at low momenta $|\mathrm{y} a| \leq 1$ the second term in Eq. (14) can be neglected. In the limit $A \rightarrow \infty, \eta \simeq e^{\frac{8 \pi \rho a^{3}}{3}}$, and this Eq. gives

$$
\tilde{\phi}_{c}^{(2)}(\mathrm{y}) \simeq \frac{n_{0}}{\pi} e^{\frac{4 \pi \rho a^{3}}{3}} \frac{\sigma \rho}{(\sigma \rho)^{2}+\mathrm{y}^{2}}
$$

which differs from the previous result in Eq. (11) by the sign in the exponent. We notice also the higher peak modification as compared with the Lorentzian form that can be obtained from Ref. [7] for a structureless fluid.

The above simple model for the response reproduces asymptotic sum rules and embodies the smearing effect of the hard-core interaction by an infinite number of spectators. Other 
models with more realistic density matrices, are now shown to share yet the same basic underlying structure and behavior.

The wave function in the product form $\Psi_{0}=\prod_{i \neq j} f\left(r_{i j}\right) / V^{A / 2}$ describes more suitably the correlations between the particles and is often used in calculations, (see for instance 《8]). The function $f\left(r_{i j}\right)$ is directly related to the pair-correlation function and tends to 1 for $r_{i j}$ larger than the correlation length. Correspondingly, the off-diagonal density matrix can be written as

$$
\rho_{A}^{(p r)}=\frac{A !}{V^{A}} \prod_{i} f\left(r_{1 i}\right) f\left(r^{\prime}{ }_{1 i}\right) \prod_{j, k \neq 1} f^{2}\left(r_{j k}\right)
$$

Now we substitute Eq. (21) into Eq. (2) and use the expansion (6), (7). The first term, $\tilde{\phi}_{1}$ is the IA, Eq. (1). For the second term one finds

$$
\tilde{\phi}_{2}^{(p r)}(\mathrm{y})=-\frac{1}{\pi A} \operatorname{Re} \int_{0}^{\infty} d x \int d^{3} r_{1} \exp (i \mathrm{y} x) E\left(\boldsymbol{r}_{1}, \boldsymbol{r}_{1}+x \hat{\boldsymbol{z}}\right)
$$

where $\hat{\boldsymbol{z}} \equiv \boldsymbol{q} / q, x=z_{1}^{\prime}-z_{1}$, and

$$
E\left(\boldsymbol{r}_{1}, \boldsymbol{r}_{1}^{\prime}\right)=\int d^{3} r_{2} \rho_{2}^{(p r)}\left(\boldsymbol{r}_{1}, \boldsymbol{r}_{2} ; \boldsymbol{r}_{1}^{\prime}, \boldsymbol{r}_{2}\right) \theta\left(a-\left|\boldsymbol{b}_{1}-\boldsymbol{b}_{2}\right|\right) \theta\left(z_{1}^{\prime}-z_{2}+w_{2}\right) \theta\left(w_{2}-z_{1}+z_{2}\right)
$$

Here $\rho_{2}$ is two-body density matrix, Eq. (5). Notice that the $\theta$-functions in Eq. (23) reduce the $r_{2}$-integration to the hatched region shown in Fig. 1.

For the third order term, $\tilde{\phi}_{3}$ also the integration over the spectator coordinates $\boldsymbol{r}_{2}, \boldsymbol{r}_{3}$, is restricted by the same hatched region (Fig. 1). While the final expression now includes the three-body density matrix it can yet be approximately reduced to one involving two-body density matrices by noticing that the function $f\left(r_{23}\right)$, describing two-body correlations in Eq. (21), is close to one except for the region of small $r_{23}$. For $x$ large enough (larger than the correlation length), $r_{23}$ will also be large for most values of the spectator coordinates, $\boldsymbol{r}_{2}, \boldsymbol{r}_{3}$, in their non-vanishing integration region (Fig. 1), and one can replace $f\left(r_{23}\right)$ by 1 in the corresponding integral for $\tilde{\phi}_{3}$. One obtains from Eqs. (5), (21)

$$
\int d^{3} r_{4} \cdots d^{3} r_{A} \rho_{A}^{(p r)}\left(\boldsymbol{r}_{1}, \ldots, \boldsymbol{r}_{A} ; \boldsymbol{r}_{1}{ }^{\prime}, \ldots, \boldsymbol{r}_{A}\right)=\frac{\rho_{2}^{(p r)}\left(\boldsymbol{r}_{1}, \boldsymbol{r}_{2} ; \boldsymbol{r}_{1}^{\prime}, \boldsymbol{r}_{2}\right) \rho_{2}^{(p r)}\left(\boldsymbol{r}_{1}, \boldsymbol{r}_{3} ; \boldsymbol{r}_{1}^{\prime}, \boldsymbol{r}_{3}\right)}{(A-1) \rho_{1}^{(p r)}\left(\boldsymbol{r}_{1} ; \boldsymbol{r}_{1}^{\prime}\right)}
$$


Using this result one gets for $\tilde{\phi}_{3}$

$$
\tilde{\phi}_{3}^{(p r)}(\mathrm{y}) \simeq \frac{1}{\pi A} \operatorname{Re} \int_{0}^{\infty} d x \int d^{3} r_{1} \exp (i \mathrm{y} x) \frac{(A-2) E^{2}\left(\boldsymbol{r}_{1}, \boldsymbol{r}_{1}+x \hat{\boldsymbol{z}}\right)}{2 \rho_{1}^{(p r)}\left(\boldsymbol{r}_{1}, \boldsymbol{r}_{1}+x \hat{\boldsymbol{z}}\right)}
$$

Applying the same approximation for higher order terms we finally obtain for the asymptotic response

$\tilde{\phi}^{(p r)}(\mathrm{y})=\frac{1}{\pi A} \operatorname{Re} \int_{0}^{\infty} d x \int d^{3} r_{1} \rho_{1}^{(p r)}\left(\boldsymbol{r}_{1} ; \boldsymbol{r}_{1}+x \hat{\boldsymbol{z}}\right) \exp (i \mathrm{y} x)\left[1-\frac{E\left(\boldsymbol{r}_{1}, \boldsymbol{r}_{1}+x \hat{\boldsymbol{z}}\right)}{(A-1) \rho_{1}^{(p r)}\left(\boldsymbol{r}_{1} ; \boldsymbol{r}_{1}+x \hat{\boldsymbol{z}}\right)}\right]^{A-1}$

Here too, similarly to Eq. (8), the FSI modifies the IA result, Eq. (1), by an additional correction factor in the integrand. If $\rho_{1}\left(\boldsymbol{r}_{1} ; \boldsymbol{r}_{1}+x \hat{\boldsymbol{z}}\right) \rightarrow$ const for $x \rightarrow \infty$, the FSI correction factor would smear the $\delta$-function peak in the response in the limit of $A \rightarrow \infty$, just in the same way as in the previous example. Notice that the integrand (26) is exact for $x \rightarrow \infty$, and therefore the smearing would always occur in the asymptotic limit.

Other treatments of the response can be compared with our asymptotic results. In 1973 Gersch and Rodriguez (GR) 10 obtained the following formula for the response, using the first cumulant term:

$$
\phi^{(G R)}(\mathrm{y})=\frac{1}{\pi \rho} \operatorname{Re} \int_{0}^{\infty} d x \rho_{1}(0 ; x) \exp \left[i \mathrm{y} x+\int d^{3} r \frac{\rho_{2}(\boldsymbol{r}, 0 ; \boldsymbol{r}+x \hat{\boldsymbol{z}}, 0)}{\rho_{1}(0 ; x)} \Omega^{(G R)}(\boldsymbol{r}, \boldsymbol{r}+x \hat{\boldsymbol{z}})\right]
$$

where

$$
\Omega^{(G R)}\left(\boldsymbol{r}, \boldsymbol{r}^{\prime}+x \hat{\boldsymbol{z}}\right)=\exp \left[-\frac{i m}{q} \int_{0}^{x} d x^{\prime}\left[v\left(\boldsymbol{r}+x^{\prime} \hat{\boldsymbol{z}}\right)-v(\boldsymbol{r}+x \hat{\boldsymbol{z}})\right]\right]-1,
$$

and $v$ is the inter-particle potential. Here translational invariance is assumed, so that $\rho_{1}\left(\boldsymbol{r}_{1} ; \boldsymbol{r}_{1}^{\prime}\right)=\rho_{1}(0 ; x)$, and $\rho_{2}\left(\boldsymbol{r}_{1}, \boldsymbol{r}_{2} ; \boldsymbol{r}_{1}^{\prime}, \boldsymbol{r}_{2}\right)=\rho_{2}(\boldsymbol{r}, 0 ; \boldsymbol{r}+x \hat{\boldsymbol{z}}, 0)$, where $\boldsymbol{r}=\boldsymbol{r}_{1}-\boldsymbol{r}_{2}$, and $\boldsymbol{r}^{\prime}=\boldsymbol{r}_{1}^{\prime}-\boldsymbol{r}_{2}$.

Although the original GR treatment was not intended for singular interactions [10], Eq. (27) can be formally applied in the case of a hard-core interaction by taking $v \rightarrow \infty$ when the particles are within their hard-core range. We consider Eq. (28) in the limit $q \rightarrow \infty$ and we take into account only the hard-core component of $v$ in Eq. (28) (since the regular component does not contribute in this limit). Then, neglecting the second term in Eq. (28) (since $\rho_{2}=0$ for $|\boldsymbol{r}+x \hat{\boldsymbol{z}}|<a$ ), one obtains 


$$
\Omega^{(G R)}(\boldsymbol{r}, \boldsymbol{r}+x \hat{\boldsymbol{z}})=-\theta(a-b) \theta(z+x+w) \theta(w-z) .
$$

Substituting Eq. (29) into Eq. (27) we find that in the limit $A \rightarrow \infty$ this equation coincides with our result, Eqs. (22), (26) (assuming translational invariance for the density matrices $\left.\rho_{1}, \rho_{2}\right)$. We thus obtain that the GR formula reproduces the asymptotic limit, Eq. (2). Since our result for the FSI correction factor is the exact one only for $x \rightarrow \infty$, the same is true for the GR formula. Also the use of the product form for the density matrix $\rho_{A}$, which takes into account only the correlation between the struck and spectator particles and neglects others [9], would reproduce Eq. (26) and therefore the GR formula (27). In this connection it is interesting to note that the same product form [9] for $\rho_{A}$, is essential in an alternative derivation of the GR result for regular interactions using a modified eikonal formula [6], [11].

Another treatment of FSI in the response function, hard-core perturbation theory (HCPT) has been proposed by Silver [12]. One readily shows that in the asymptotic limit HCPT and GR lead to different results [12]. Indeed, HPCT response can be written in the form of Eq. (27), where

$$
\Omega^{(H C P T)}=-\theta(a-b) \theta(z+x)
$$

(which was used together with $\rho_{2} / \rho^{2}=\theta(r-a)$ in the comparison with GR), is different from $\Omega^{(G R)}$ as given by Eq. (29). It thus follows from the above consideration that HCPT does not reproduce the asymptotic limit for $x \rightarrow \infty$.

Next, we discuss the application of our results to the response of Fermi systems interacting through a hard-core. Weinstein and Negele [13] studied numerically such systems. They considered the asymptotic limit for the response function and found considerable deviations from the IA at $|\mathrm{y}|>k_{F}$, where $k_{F}$ is the Fermi momentum. We now derive from our approach a simple analytical expression for the asymptotic response of a Fermi gas with hard-core interaction, at small $|\mathrm{y}|$.

Consider the limit of $4 \pi \rho a^{3} / 3 \ll 1$ (the hard-core volume is small with respect to the volume per particle). In this case the Fermi gas momentum distribution, $n_{F}(p)=\frac{6 \pi^{2}}{k_{F}^{3}} \theta\left(k_{F}-\right.$ 
$p$ ), is not essentially modified by hard-core interactions. However, the hard-core inevitably generates high momentum components in $n(p)$. Therefore the application of our approach will be reliable in the region $|\mathrm{y}|<k_{F}$. The corresponding one-body density matrix is

$$
\rho_{1 F}(0 ; x)=3 \rho \frac{j_{1}\left(k_{F} x\right)}{k_{F} x} .
$$

We can consequently write the Fermi gas $A$-particle density matrix as

$$
\rho_{A F}=\frac{A !}{\rho V^{A}} \rho_{1 F}(x) .
$$

Substituting Eq. (32) into Eq. (2) and taking the limit $A \rightarrow \infty$ we find

$$
\begin{aligned}
\tilde{\phi}_{F}(\mathrm{y}) & =\frac{3}{\pi} \operatorname{Re} \int_{0}^{\infty} \frac{j_{1}\left(k_{F} x\right)}{k_{F} x} \exp (i \mathrm{y} x-\rho \sigma x) d x \\
& =\frac{3}{4 \pi k_{F}^{3}} \operatorname{Im}\left\{\left[(\mathrm{y}+i \sigma \rho)^{2}-k_{F}^{2}\right] \ln \frac{\mathrm{y}+i \sigma \rho+k_{F}}{\mathrm{y}+i \sigma \rho-k_{F}}\right\}-\frac{3 \sigma \rho}{2 \pi k_{F}^{2}} .
\end{aligned}
$$

Notice that in the limit $\sigma=\pi a^{2} \rightarrow 0$, Eq. (33) gives the well known IA result for the Fermi gas response, $\phi_{F}^{I A}(\mathrm{y})=3\left(k_{F}^{2}-\mathrm{y}^{2}\right) \theta\left(k_{F}-|\mathrm{y}|\right) / 4 k_{F}^{3}$.

In Fig. 2 we plot the resulting Fermi gas response, Eq. (33), for different values of the hard-core radius. We take nuclear matter as an example with $k_{F}=1.33 \mathrm{fm}^{-1}$ and $\rho=2 k_{F}^{3} / 3 \pi^{2}$. The solid line shows the IA result $(a=0)$, and the dot-dashed and dashed lines correspond to $a=0.5 \mathrm{fm}$ and $a=0.8 \mathrm{fm}$, respectively. The ratio of the hard-core volume to $1 / \rho$ is 0.084 for $a=0.5 \mathrm{fm}$, and 0.34 for $a=0.8 \mathrm{fm}$. Therefore the use of the Fermi momentum distribution in Eq. 32 is still approximately correct. We find that the response considerably deviates from the IA. One also notices the disappearance of the singularity in the response at $|\mathrm{y}|=k_{F}$ due to hard-core FSI.

It is also interesting to note the sizable effect from the hard-core FSI in the region of the quasi-elastic peak. For $a=0.5 \mathrm{fm}$ the response is reduced by about $10 \%$. Although such a reduction has been the result of a pure hard-core FSI, a similar effect of FSI would be also expected for a strong repulsive interaction, provided the potential is much larger than the energy of the recoil particle for distances smaller than $a$. 
In summary, we have investigated the effect of hard-core interactions on the response function obtained from inclusive scattering, around the quasielastic peak. Relevant models of the density matrix describing systems of particles interacting through a hard-core were constructed. By using them the response was shown to be modified and smeared as compared with the otherwise expected IA form in the asymptotic limit, both for bosons and fermions. New analytical formulae were obtained for the response of these systems reproducing quite generally the asymptotic behaviour of the response and that satisfy the asymptotic sum rules.

\section{Acknowledgements}

We thank A. S. Rinat for discussions and suggestions in the writing. 


\section{REFERENCES}

[1] S. A. Gurvitz, A. S. Rinat, and R. Rosenfelder, Phys. Rev. C 40 (1989) 1363.

[2] M. Born and E. Wolf, Principles of Optics (Pergamon, Oxford, 1970), Chap. 3.

[3] P. C. Hohenberg and P. M. Platzman, Phys. Rev. 152 (1966) 198.

[4] A. S. Rinat and M. F. Taragin, Phys. Rev. B 41 (1990) 4247.

[5] C. Carraro, A. S. Rinat, Phys. Rev. B 45 (1992) 2945.

[6] J. Besprosvany, Phys. Rev. B 46 (1992) 14226.

[7] P. M. Platzman, N. Tzoar, Phys. Rev. B 30 (1984) 6397.

[8] C. Carraro and S.E. Koonin, Phys. Rev. Lett. 65 (1990) 2792.

[9] A. Kohama, K. Yazaki and R. Seki, Nucl. Phys. A 536, (1992) 716.

[10] H. A. Gersch and L. J. Rodriguez, Phys. Rev. A 8 (1973) 905.

[11] J. Besprosvany, Phys. Rev. B 43 (1991) 10070.

[12] R. N. Silver, Phys. Rev. B 38 (1988) 2283.

[13] J. J. Weinstein and J. W. Negele, Phys. Rev. Lett. 49 (1982) 1016. 


\section{Figure Captions.}

Fig 1. Hard-core shadow configuration in space $\left(\boldsymbol{r}_{i}\right)$ for the spectator particle, produced by the Green's function in Eq. (2) (hatched region). Square-hatched circles show the regions of the hard-cores' interception.

Fig. 2. Asymptotic response for a Fermi-gas in the impulse approximation (solid), and with hard-core final state interactions corrections with core radius $a=0.5 \mathrm{fm}$ (dot-dashes), and $a=0.8 \mathrm{fm}$ (dashes). 